\title{
Erratum to: LPS response pattern of inflammatory adipokines in an in vitro 3T3-L1 murine adipocyte model
}

\author{
Salvatore Chirumbolo • Guido Franceschetti - Elena Zoico • \\ Clara Bambace $\cdot$ Luciano Cominacini $\cdot$ Mauro Zamboni
}

Published online: 21 March 2014

(C) Springer Basel 2014

\section{Erratum to: Inflamm Res}

DOI 10.1007/s00011-014-0721-9

The original publication of this article unfortunately contained an error in Tables 1, 2 and Fig. 3. In both tables published, the last column on the right [LPS doses $(\mathrm{ng} / \mathrm{ml})$ ] does not include the complete data for the rows. In Fig. 3, parts of the panel a and d [(a) left $y$-axis is IL-6 (ng/ml); (d) left $y$-axis is MIP-1beta/CCL4 (pg/ml)] appear hidden.

The corrected tables and figure are given below.

Responsible Editor: John Di Battista.

The online version of the original article can be found under doi:10.1007/s00011-014-0721-9.

S. Chirumbolo $(\bowtie) \cdot$ G. Franceschetti - E. Zoico •

C. Bambace $\cdot$ M. Zamboni

Unit of Geriatry, Department of Medicine,

University of Verona, Verona, Italy

e-mail: salvatore.chirumbolo@univr.it

L. Cominacini

Unit of Internal Medicine, Department of Medicine,

University of Verona, Verona, Italy 
Table 1 Effects of E. coli lipopolysaccharide (LPS) on 3T3-L1 adipokine gene expression at different times

\begin{tabular}{|c|c|c|c|c|c|}
\hline \multirow[t]{2}{*}{ Target gene ${ }^{\mathrm{a}}$} & \multicolumn{4}{|c|}{ Time course (hours) mean $\pm \mathrm{SD}$ and $p$ values* } & \multirow[t]{2}{*}{ LPS doses $(\mathrm{ng} / \mathrm{ml})$} \\
\hline & 4 & 8 & 16 & 24 & \\
\hline \multirow[t]{6}{*}{ IL-6 } & $0.95 \pm 0.07$ & $1.04 \pm 0.08$ & $1.07 \pm 0.08$ & $1.02 \pm 0.13$ & 0 \\
\hline & $0.49 \pm 0.01$ & $4.95 \pm 0.04(p<0.01)$ & $2.95 \pm 0.91(p<0.01)$ & $3.82 \pm 0.01(p<0.05)$ & 1 \\
\hline & $1.03 \pm 0.06$ & $8.52 \pm 0.02(p<0.01)$ & $7.41 \pm 1.34(p<0.001)$ & $9.36 \pm 0.15(p<0.01)$ & 10 \\
\hline & $1.01 \pm 0.02$ & $8.49 \pm 0.05(p<0.05)$ & $10.01 \pm 0.35(p<0.001)$ & $8.71 \pm 0.34(p<0.001)$ & 100 \\
\hline & $1.62 \pm 0.03$ & $6.98 \pm 0.04(p<0.05)$ & $16.58 \pm 0.18(p<0.001)$ & $20.21 \pm 0.42(p<0.001)$ & 1,000 \\
\hline & $2.27 \pm 0.03$ & $8.50 \pm 0.14(p<0.01)$ & $14.37 \pm 1.07(p<0.001)$ & $15.31 \pm 1.46(p<0.001)$ & 10,000 \\
\hline \multirow[t]{6}{*}{ TNF- $\alpha$} & $0.98 \pm 0.08$ & $0.95 \pm 0.07$ & $1.00 \pm 0.01$ & $1.00 \pm 0.01$ & 0 \\
\hline & $1.04 \pm 0.06$ & $2.24 \pm 0.67(p<0.01)$ & $1.61 \pm 0.03(p<0.05)$ & $15.11 \pm 0.04(p<0.001)$ & 1 \\
\hline & $1.06 \pm 0.07$ & $1.27 \pm 0.23(p<0.01)$ & $3.07 \pm 0.01(p<0.01)$ & $15.76 \pm 0.05(p<0.001)$ & 10 \\
\hline & $0.98 \pm 0.10$ & $3.15 \pm 0.92(p<0.01)$ & $3.21 \pm 0.04(p<0.01)$ & $16.41 \pm 0.15(p<0.01)$ & 100 \\
\hline & $1.01 \pm 0.04$ & $10.23 \pm 0.31(p<0.001)$ & $11.18 \pm 0.41(p<0.05)$ & $19.21 \pm 0.25(p<0.001)$ & 1,000 \\
\hline & $0.98 \pm 0.09$ & $9.67 \pm 1.88(p<0.001)$ & $12.21 \pm 0.19(p<0.001)$ & $25.01 \pm 0.28(p<0.001)$ & 10,000 \\
\hline \multirow[t]{6}{*}{ MIP-1 $\alpha /$ CCL3 } & $1.00 \pm 0.01$ & $1.02 \pm 0.04$ & $1.00 \pm 0.01$ & $1.02 \pm 0.06$ & 0 \\
\hline & $0.26 \pm 0.01$ & $6.83 \pm 0.40(p<0.01)$ & $0.09 \pm 0.01$ & $0.41 \pm 0.09$ & 1 \\
\hline & $0.47 \pm 0.04$ & $13.56 \pm 0.27(p<0.001)$ & $0.04 \pm 0.01$ & $0.24 \pm 0.04$ & 10 \\
\hline & $0.64 \pm 0.15$ & $19.64 \pm 1.29(p<0.001)$ & $0.96 \pm 0.04$ & $0.49 \pm 0.02$ & 100 \\
\hline & $1.12 \pm 0.14$ & $27.57 \pm 0.67(p<0.001)$ & $4.17 \pm 0.16(p<0.01)$ & $0.62 \pm 0.13$ & 1,000 \\
\hline & $1.43 \pm 0.10$ & $30.52 \pm 2.12(p<0.001)$ & $3.95 \pm 0.46(p<0.001)$ & $0.11 \pm 0.02$ & 10,000 \\
\hline \multirow[t]{6}{*}{ CXCR4 } & $0.96 \pm 0.07$ & $0.99 \pm 0.01$ & $1.00 \pm 0.09$ & $0.89 \pm 0.10$ & 0 \\
\hline & $1.09 \pm 0.13$ & $0.96 \pm 0.07$ & $1.02 \pm 0.01$ & $0.99 \pm 0.07$ & 1 \\
\hline & $0.99 \pm 0.02$ & $1.97 \pm 0.67$ & $1.24 \pm 0.02$ & $5.71 \pm 0.40(p<0.001)$ & 10 \\
\hline & $0.98 \pm 0.08$ & $3.49 \pm 0.26(p<0.05)$ & $1.67 \pm 0.21$ & $6.16 \pm 0.35(p<0.001)$ & 100 \\
\hline & $1.02 \pm 0.05$ & $6.19 \pm 0.60(p<0.01)$ & $1.55 \pm 0.02$ & $3.67 \pm 0.38(p<0.001)$ & 1,000 \\
\hline & $1.07 \pm 0.12$ & $6.07 \pm 0.36(p<0.01)$ & $1.74 \pm 0.06(p<0.05)$ & $2.25 \pm 0.26(p<0.01)$ & 10,000 \\
\hline \multirow[t]{6}{*}{ MIP-1 $\beta / C C L 4$} & $0.96 \pm 0.07$ & $1.03 \pm 0.07$ & $0.99 \pm 0.01$ & $0.97 \pm 0.17$ & 0 \\
\hline & $0.66 \pm 0.03$ & $2.90 \pm 0.04(p<0.01)$ & $1.09 \pm 0.16$ & $0.71 \pm 0.05$ & 1 \\
\hline & $0.64 \pm 0.02$ & $3.59 \pm 0.23(p<0.01)$ & $1.13 \pm 0.22$ & $049 \pm 0.01$ & 10 \\
\hline & $0.72 \pm 0.09$ & $4.09 \pm 0.40(p<0.001)$ & $1.13 \pm 0.14$ & $0.58 \pm 0.01$ & 100 \\
\hline & $1.16 \pm 0.13$ & $6.24 \pm 0.27(p<0.001)$ & $1.09 \pm 0.16$ & $0.10 \pm 0.01$ & 1,000 \\
\hline & $1.43 \pm 0.11$ & $2.22 \pm 0.13(p<0.001)$ & $1.13 \pm 0.22$ & $0.01 \pm 0.01$ & 10,000 \\
\hline \multirow[t]{6}{*}{ CXCL12/SDF1 } & $0.96 \pm 0.07$ & $0.95 \pm 0.07$ & $0.95 \pm 0.06$ & $0.99 \pm 0.02$ & 0 \\
\hline & $0.15 \pm 0.01$ & $1.24 \pm 0.02$ & $2.95 \pm 0.10(p<0.05)$ & $3.07 \pm 0.05(p<0.05)$ & 1 \\
\hline & $0.06 \pm 0.01$ & $1.05 \pm 0.03$ & $3.49 \pm 0.12(p<0.05)$ & $3.50 \pm 0.02(p<0.001)$ & 10 \\
\hline & $0.26 \pm 0.01$ & $1.05 \pm 0.05$ & $3.14 \pm 0.11(p<0.01)$ & $3.41 \pm 0.03(p<0.01)$ & 100 \\
\hline & $0.31 \pm 0.01$ & $1.11 \pm 0.02$ & $3.31 \pm 0.07(p<0.05)$ & $3.23 \pm 0.06(p<0.01)$ & 1,000 \\
\hline & $0.17 \pm 0.01$ & $1.68 \pm 0.04(p<0.05)$ & $2.52 \pm 0.14(p<0.01)$ & $2.64 \pm 0.03(p<0.001)$ & 10,000 \\
\hline \multirow[t]{6}{*}{ IL-10 } & $0.95 \pm 0.07$ & $0.96 \pm 0.18$ & $0.96 \pm 0.07$ & $1.01 \pm 0.04$ & 0 \\
\hline & $1.02 \pm 0.07$ & $0.82 \pm 0.02$ & $2.65 \pm 0.07(p<0.01)$ & $4.19 \pm 0.16(p<0.001)$ & 1 \\
\hline & $0.96 \pm 0.06$ & $1.71 \pm 0.02(p<0.05)$ & $4.10 \pm 0.11(p<0.01)$ & $2.20 \pm 0.06(p<0.001)$ & 10 \\
\hline & $1.02 \pm 0.06$ & $2.18 \pm 0.08(p<0.01)$ & $1.23 \pm 0.03$ & $1.37 \pm 0.06$ & 100 \\
\hline & $0.98 \pm 0.11$ & $0.89 \pm 0.03$ & $1.12 \pm 0.01$ & $1.24 \pm 0.02$ & 1,000 \\
\hline & $1.03 \pm 0.06$ & $0.99 \pm 0.01$ & $1.02 \pm 0.04$ & $1.08 \pm 0.02$ & 10,000 \\
\hline
\end{tabular}

* Statistics with Wilcoxon-Mann-Whitney $U$ test. Test significant for $p<0.05$. Significance compared to resting condition $(0$ LPS) for each time point

${ }^{\mathrm{a}}$ Data are expressed as normalized expression folds 
Table 2 Effects of E. coli lipopolysaccharide (LPS) on 3T3-L1 protein release at different times

\begin{tabular}{|c|c|c|c|c|c|}
\hline \multirow[t]{2}{*}{ Protein } & \multicolumn{4}{|c|}{ Time course (hours) mean \pm SD and $p$ values* } & \multirow{2}{*}{$\begin{array}{l}\text { LPS } \\
\text { doses } \\
(\mathrm{ng} / \mathrm{ml})\end{array}$} \\
\hline & 4 & 8 & 16 & 24 & \\
\hline \multirow[t]{6}{*}{ IL-6 (ng/ml) } & $0.10 \pm 0.04$ & $1.00 \pm 0.04$ & $15.12 \pm 0.17(p<0.001)$ & $89.86 \pm 1.98(p<0.001)$ & 0 \\
\hline & $0.20 \pm 0.01$ & $3.15 \pm 0.13(p<0.01)$ & $25.25 \pm 0.24(p<0.001)$ & $95.89 \pm 1.88(p<0.001)$ & 1 \\
\hline & $0.58 \pm 0.04$ & $6.77 \pm 0.22(p<0.001)$ & $31.67 \pm 1.00(p<0.001)$ & $133.44 \pm 7.72(p<0.001)$ & 10 \\
\hline & $1.03 \pm 0.06$ & $7.61 \pm 0.31(p<0.001)$ & $40.19 \pm 1.35(p<0.001)$ & $121.78 \pm 3.48(p<0.001)$ & 100 \\
\hline & $1.94 \pm 0.06(p<0.05)$ & $8.41 \pm 0.14(p<0.001)$ & $64.69 \pm 0.65(p<0.001)$ & $194.10 \pm 5.6(p<0.001) 1$ & 1,000 \\
\hline & $2.98 \pm 0.23(p<0.01)$ & $10.73 \pm 0.61(p<0.001)$ & $56.65 \pm 2.22(p<0.001)$ & $175.57 \pm 0.88(p<0.001)$ & 10,000 \\
\hline \multirow[t]{6}{*}{$\mathrm{TNF}-\alpha(\mathrm{ng} / \mathrm{ml})$} & $0.34 \pm 0.45$ & $1.07 \pm 0.06(p<0.05)$ & $10.51 \pm 0.17(p<0.001)$ & $86.63 \pm 0.86(p<0.001)$ & 0 \\
\hline & $0.11 \pm 0.01$ & $2.99 \pm 0.01(p<0.001)$ & $16.45 \pm 0.42(p<0.001)$ & $139.24 \pm 3.84(p<0.001)$ & 1 \\
\hline & $0.21 \pm 0.02$ & $3.11 \pm 0.40(p<0.01)$ & $32.10 \pm 3.05(p<0.001)$ & $148.66 \pm 0.47(p<0.001)$ & 10 \\
\hline & $0.40 \pm 0.01$ & $5.28 \pm 0.06(p<0.001)$ & $45.33 \pm 0.87(p<0.01)$ & $145.69 \pm 11.34(p<0.001)$ & 100 \\
\hline & $0.58 \pm 0.01$ & $8.76 \pm 0.78(p<0.001)$ & $76.41 \pm 0.98(p<0.001)$ & $170.70 \pm 0.95(p<0.001)$ & 1,000 \\
\hline & $0.65 \pm 0.02$ & $8.61 \pm 0.06$ & $82.75 \pm 3.23(p<0.001)$ & $180.62 \pm 1.06(p<0.001)$ & 10,000 \\
\hline \multirow{6}{*}{$\begin{array}{l}\text { MIP-1 } \alpha / \text { CCL3 (pg/ } \\
\quad \mathrm{ml})\end{array}$} & Not detectable & $19.29 \pm 0.87(p<0.001)$ & $108.29 \pm 0.66(p<0.001)$ & $140.08 \pm 1.25(p<0.001)$ & 0 \\
\hline & $1.02 \pm 0.08$ & $24.17 \pm 0.83(p<0.001)$ & $116.22 \pm 2.62(p<0.001)$ & $123.05 \pm 1.37(p<0.001)$ & 1 \\
\hline & $3.35 \pm 0.08(p<0.001)$ & $38.46 \pm 1.62(p<0.001)$ & $116.23 \pm 2.60(p<0.001)$ & $133.34 \pm 9.19(p<0.001)$ & 10 \\
\hline & $4.04 \pm 0.08(p<0.001)$ & $46.43 \pm 1.49(p<0.001)$ & $136.49 \pm 5.44(p<0.001)$ & $113.51 \pm 7.93(p<0.001)$ & 100 \\
\hline & $4.65 \pm 0.13(p<0.001)$ & $73.26 \pm 0.65(p<0.001)$ & $161.42 \pm 6.91(p<0.001)$ & $116.78 \pm 6.84(p<0.001)$ & 1,000 \\
\hline & $8.23 \pm 0.17(p<0.01)$ & $96.45 \pm 0.65(p<0.001)$ & $170.10 \pm 1.37(p<0.001)$ & $100.72 \pm 7.26(p<0.001)$ & 10,000 \\
\hline \multirow{7}{*}{$\begin{array}{l}\text { MIP-1 } \beta / \text { CCL4 (pg/ } \\
\quad \mathrm{ml})\end{array}$} & Not detectable & $7.48 \pm 0.67(p<0.001)$ & $14.74 \pm 1.18(p<0.001)$ & $8.45 \pm 0.39$ & 0 \\
\hline & $0.15 \pm 0.03$ & $15.59 \pm 0.79(p<0.001)$ & $13.27 \pm 0.2(p<0.001) 9$ & $7.57 \pm 0.10(p<0.01)$ & 1 \\
\hline & $1.97 \pm 0.05$ & $24.03 \pm 3.36(p<0.001)$ & $12.51 \pm 0.47(p<0.001)$ & $7.00 \pm 0.01$ & 10 \\
\hline & $3.19 \pm 0.02$ & $37.21 \pm 1.63(p<0.01)$ & $13.53 \pm 1.11(p<0.001)$ & $5.71 \pm 0.26(p<0.001)$ & 100 \\
\hline & $5.23 \pm 0.09$ & $50.40 \pm 1.34(p<0.001)$ & $12.69 \pm 0.84(p<0.001)$ & $3.55 \pm 0.28(p<0.001)$ & 1,000 \\
\hline & $6.73 \pm 0.18$ & $50.37 \pm 0.43(p<0.001)$ & $12.37 \pm 0.56(p<0.01)$ & $1.26 \pm 0.06(p<0.001)$ & 10,000 \\
\hline & $(p<0.001)$ & & & & \\
\hline \multirow{6}{*}{$\begin{array}{l}\text { CXCL12/SDF1 } \\
\text { (ng/ml) }\end{array}$} & $1.01 \pm 0.02$ & $2.04 \pm 0.06(p<0.01)$ & $5.34 \pm 0.11(p<0.001)$ & $8.48 \pm 0.50(p<0.001)$ & 0 \\
\hline & $0.59 \pm 0.1$ & $2.56 \pm 0.10(p<0.05)$ & $7.37 \pm 0.52(p<0.001)$ & $11.67 \pm 0.10(p<0.001)$ & 1 \\
\hline & $0.60 \pm 0.01$ & $2.96 \pm 0.07(p<0.001)$ & $8.41 \pm 0.36(p<0.001)$ & $11.88 \pm 0.97(p<0.001)$ & 10 \\
\hline & $0.51 \pm 0.05$ & $3.04 \pm 0.07(p<0.001)$ & $9.52 \pm 0.07(p<0.001)$ & $13.15 \pm 0.06(p<0.001)$ & 100 \\
\hline & $0.44 \pm 0.04$ & $3.37 \pm 0.12(p<0.001)$ & $7.92 \pm 0.13(p<0.001)$ & $11.03 \pm 0.06(p<0.001)$ & 1,000 \\
\hline & $0.53 \pm 0.03$ & $4.20 \pm 0.15(p<0.001)$ & $7.49 \pm 0.12(p<0.001)$ & $9.04 \pm 0.07(p<0.001)$ & 10,000 \\
\hline \multirow[t]{6}{*}{ IL-10 (ng/ml) } & $0.86 \pm 0.12$ & $6.81 \pm 0.21(p<0.001)$ & $15.62 \pm 0.99(p<0.001)$ & $11.27 \pm 0.43(p<0.001)$ & 0 \\
\hline & $1.54 \pm 0.11$ & $6.84 \pm 0.68(p<0.001)$ & $21.29 \pm 0.75(p<0.001)$ & $38.00 \pm 0.54(p<0.001)$ & 1 \\
\hline & $1.86 \pm 0.12$ & $8.60 \pm 0.57(p<0.001)$ & $33.97 \pm 0.28(p<0.001)$ & $31.37 \pm 0.57(p<0.001)$ & 10 \\
\hline & $1.15 \pm 0.06$ & $10.03 \pm 0.17(p<0.001)$ & $34.48 \pm 1.50(p<0.001)$ & $19.47 \pm 0.59(p<0.001)$ & 100 \\
\hline & $0.91 \pm 0.13$ & $8.47 \pm 0.45(p<0.001)$ & $28.62 \pm 0.67(p<0.001)$ & $12.78 \pm 1.18(p<0.001)$ & 1,000 \\
\hline & $0.56 \pm 0.08$ & $7.45 \pm 0.11(p<0.001)$ & $16.04 \pm 0.62$ & $10.59 \pm 0.55(p<0.001)$ & 10,000 \\
\hline
\end{tabular}

* Statistics with Wilcoxon-Mann-Whitney $U$ test. Test significant for $p<0.05$. Significance compared to resting condition (0 LPS) for each time point 

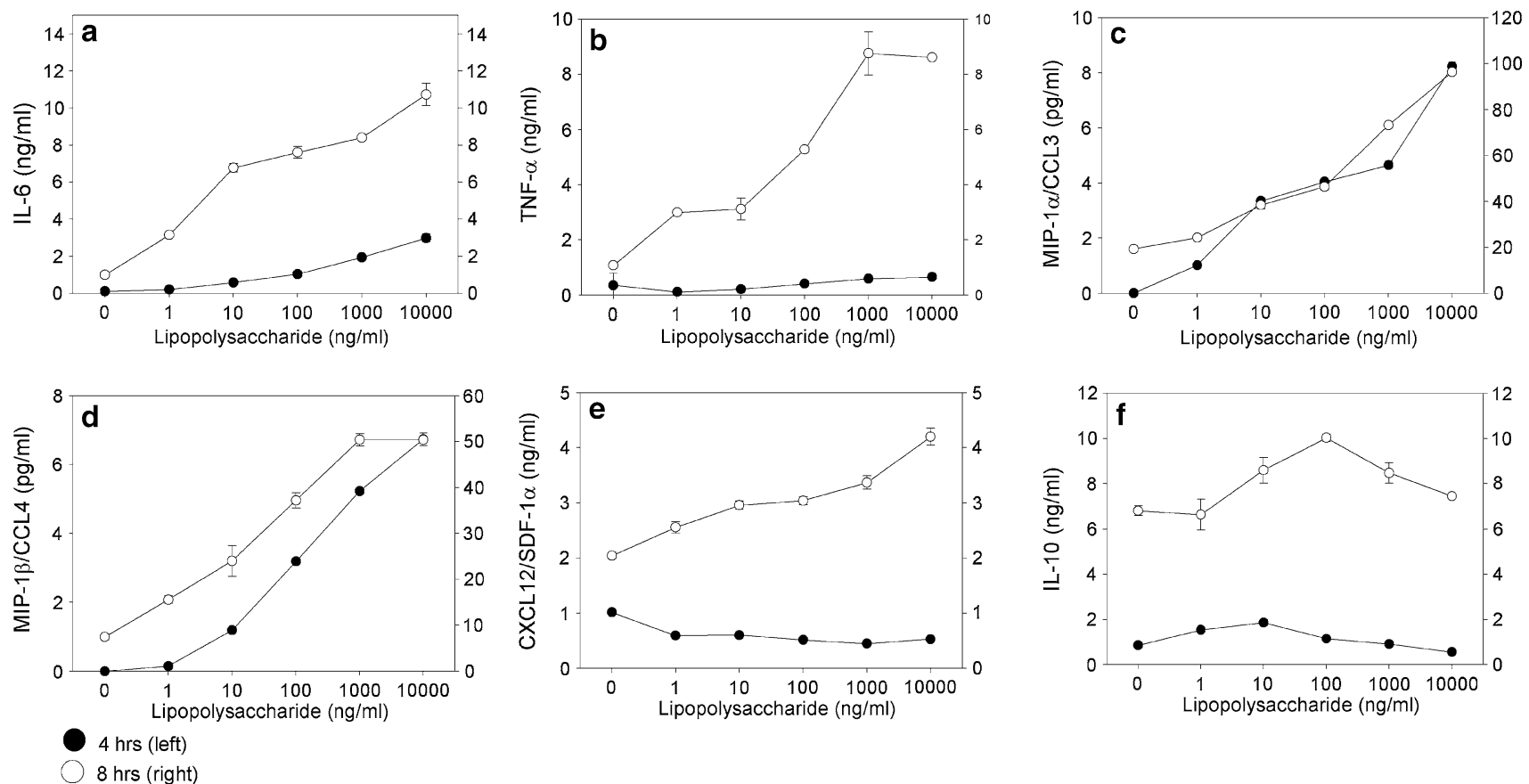

Fig. 3 Adipokine production evaluated by ELISA of the investigated adipokines following $4 \mathrm{~h}$ (black) and $8 \mathrm{~h}$ (white) incubation with the indicated LPS doses (ng/ml), For further information see text. Data are mean \pm SD of three separate assays 